\title{
On the Convexity of Precedence Sequencing Games
}

\author{
Herbert Hamers ${ }^{\mathrm{a}}$, Flip Klijn ${ }^{\mathrm{b}, 1}$, Bas van Velzen ${ }^{\mathrm{a}}$ \\ ${ }^{\mathrm{a}}$ CentER and Department of Econometrics and Operational Research, \\ Tilburg University, The Netherlands \\ ${ }^{\mathrm{b}} \mathrm{CODE}$ and Departament d'Economia i d'Història Econòmica, \\ Universitat Autònoma de Barcelona, Spain
}

\begin{abstract}
In this paper we study a class of cooperative sequencing games that arise from one-machine sequencing situations in which chain precedence relations are imposed on the jobs. It is shown that these sequencing games are convex.
\end{abstract}

Keywords: Cooperative games, sequencing situations

\section{Introduction}

In operations research, sequencing situations are characterized by a finite number of jobs lined up in front of one (or more) machine(s) that have to be processed on the machine(s). A single decision maker wants to determine a processing order of the jobs that minimizes a cost criterion and takes into account possible restriction on the jobs (e.g. due dates, precedence constraints, etc.) This single decision maker problem can be transformed into a multiple decision maker problem by taking agents into account who own at least one job. In such a model a group of agents (coalition) can save costs by cooperation. For the determination of the maximal cost savings of a coalition one has to solve the combinatorial optimization problem corresponding to this coalition.

This approach has been taken first in Curiel et al. (1989). They introduce sequencing games, which arise from one-machine sequencing situations, and showed that these games are convex, and thus, balanced. Moreover, they introduce and characterize an allocation rule that divides the maximal cost savings that can be obtained by complete cooperation.

The paper by Curiel et al. (1989) has inspired researchers to study the interaction between scheduling theory and cooperative game theory. Hamers et al. (1996) and Van Velzen and Hamers (2002) investigate the class of sequencing situations as in considered Curiel et al. (1989). The first paper focuses on the

\footnotetext{
${ }^{1}$ Corresponding author. CODE and Departament d'Economia i d'Història Econòmica, Universitat Autònoma de Barcelona, Edifici B, 08193 Bellaterra, Spain. Tel. (34) 93581 1720; Fax. (34) 93581 2012; e-mail: fklijn@pareto.uab.es. This author's research has been supported by a Marie Curie Fellowship of the European Community programme "Improving Human Research Potential and the Socio-economic Knowledge Base” under contract number HPMF-CT-2001-01232.
} 
structure of a subset of the core, the split core, and the second paper introduces new classes of balanced sequencing games.

Van den Nouweland et al. (1992), Hamers et al. (1999) and Calleja et al. (2002) investigate sequencing games that arise from multiple-machine sequencing situations. These papers focus on the balancedness of the related sequencing games.

In the class of sequencing situations considered in Curiel et al. (1989) no restrictions like ready times or due dates are imposed on the jobs. Hamers et al. (1995) included ready times (or release dates) on the one-machine sequencing situations considered by Curiel et al. (1989). In this case the corresponding sequencing games are balanced, but are not necessarily convex. For a special subclass, however, convexity could be established. Similar results are also obtained in Borm et al. (2002), in which due dates are imposed on the jobs.

This paper is in the same line as Hamers et al. (1995) and Borm et al. (2002). Here, precedence relations are imposed on the job in one-machine sequencing situations. Precedence relations prescribe an order in which jobs have to be processed. More specifically, some jobs can only be processed if some other job(s) have already been processed. In practice many examples can be found where precedence relations play a role. For example, scheduling programs on a computer. In many cases one program needs the output of another program as input data. Another situation where precedence relations are involved is in the manufacturing of a car. Before you can paint the car you need to have the chassis, before you can place the wheels you need already the axles, etc. In this paper we establish a convexity result for sequencing games that arise from sequencing situations in which chain precedence relations are involved.

There are several arguments to ask for convexity. Convex (or supermodular) games are known to have nice properties, in the sense that some solutions concepts for these games coincide and others have intuitive descriptions. For example, for convex games the core is the convex hull of all marginal vectors (cf. Shapley (1971) and Ichiishi (1981)), and, as a consequence, the Shapley value is the barycentre of the core (Shapley (1971)). Moreover, the bargaining set and the core coincide, the kernel coincides with the nucleolus (Maschler et al. (1972)) and the $\tau$-value can easily be calculated (Tijs (1981)).

The paper is organized as follows. In Section 2 we introduce one-machine precedence sequencing situations and the related precedence sequencing games. We present our convexity result in Section 3. In the Appendix we prove rather technical lemmata needed for the convexity result of Section 3.

\section{Precedence sequencing situations and games}

In this section we describe a one-machine sequencing situation in which precedence relations hold for the jobs. Moreover, we define the corresponding sequencing games.

In a one-machine precedence sequencing situation there is a queue of agents, each with one job, before a machine (counter). Each agent (player) has to process his job on the machine. The finite set of agents is denoted by $N$, and its cardinality by $|N|=n$. A processing order is defined by a bijection $\sigma: N \rightarrow\{1, \ldots, n\}$. Specifically, $\sigma(i)=k$ means that player $i$ is in position $k$. A precedence relation $\mathcal{P}$ on the jobs of the players is defined as follows: if $(i, j) \in \mathcal{P}$ then the job of player $i$ has to precede the job of player $j$. Obviously, for any $\mathcal{P}$ we have that if $(i, j) \in \mathcal{P}$ then $(j, i) \notin \mathcal{P}$. A processing order is called feasible with respect to $\mathcal{P}$ if for all $(i, j) \in \mathcal{P}$ it holds that $i$ precedes $j$ in that order. The set of all feasible processing orders of $N$ with respect to $\mathcal{P}$ is denoted by $\Pi(N, \mathcal{P})$. The processing time $p_{i}$ of the job of agent $i$ is the time the machine takes to handle this job. We assume that every agent has a linear cost function $c_{i}:[0, \infty) \rightarrow \mathbb{R}_{+}$defined by $c_{i}(t)=\alpha_{i} t$ with $\alpha_{i}>0$. Further it is assumed that 
there is an initial feasible order $\sigma_{0}: N \rightarrow\{1, \ldots, n\}$ on the jobs of the players before the processing of the machine starts.

A precedence sequencing situation as described above is denoted by $\left(N, \mathcal{P}, \sigma_{0}, p, \alpha\right)$, where $N$ is the set of $n$ players, $\mathcal{P}$ the set of precedence relations, $\sigma_{0}: N \rightarrow\{1, \ldots, n\}$ the initial order, $p=\left(p_{i}\right)_{i \in N} \in$ $\mathbb{R}_{+}^{N}$ the vector representing the processing times and $\alpha=\left(\alpha_{i}\right)_{i \in N} \in \mathbb{R}_{+}^{N}$ the vector denoting the cost coefficients.

For an order $\sigma$ the set of predecessors of player $i \in N$ is $\operatorname{Pr}(\sigma, i)=\{j \mid \sigma(j)<\sigma(i)\}$. Then the completion time $C(\sigma, i)$ of the job of agent $i$ with respect to some feasible order $\sigma$ is equal to $p_{i}+\sum_{j \in \operatorname{Pr}(\sigma, i)} p_{j}$. The total costs $c_{\sigma}(S)$ of a coalition $S \subseteq N$ is given by

$$
c_{\sigma}(S)=\sum_{i \in S} \alpha_{i}(C(\sigma, i))
$$

The (maximal) cost savings of a coalition $S$ depend on the precedence relation $\mathcal{P}$ and the set of admissible orders of this coalition. We call a processing order $\sigma \in \Pi(N, \mathcal{P})$ admissible for $S$ with respect to the initial order if it satisfies the following condition:

$$
\operatorname{Pr}\left(\sigma_{0}, j\right)=\operatorname{Pr}(\sigma, j) \text { for all } j \in N \backslash S \text {. }
$$

This condition implies that the completion time of each agent outside the coalition $S$ is equal to his completion time in the initial order, and that the agents of $S$ are not allowed to jump over players outside $S$. The set of admissible orders for a coalition $S$ is denoted by $\Sigma(S, \mathcal{P})$.

Given a precedence sequencing situation $\left(N, \mathcal{P}, \sigma_{0}, p, \alpha\right)$ the corresponding precedence sequencing game is defined in such a way that the worth of a coalition $S$ is equal to the maximal cost savings the coalition can achieve by means of an admissible order. Formally we have for any $S \subseteq N, S \neq \emptyset$ that

$$
v(S)=\max _{\sigma \in \Sigma(S, \mathcal{P})}\left\{\sum_{i \in S}\left(\alpha_{i} C\left(\sigma_{0}, i\right)\right)-\sum_{i \in S}\left(\alpha_{i} C(\sigma, i)\right)\right\}
$$

A coalition $S$ is called connected with respect to $\sigma_{0}$ if for all $i, j \in S$ and $k \in N, \sigma_{0}(i)<\sigma_{0}(k)<\sigma_{0}(j)$ implies $k \in S$. A connected coalition $S \subseteq T$ is a component of $T$ if $i \in T \backslash S$ implies that $S \cup\{i\}$ is not connected. The components of $T$ form a partition of $T$, denoted by $T / \sigma_{0}$. The definition of an admissible order of a coalition $S$ says the players of $S$ are not allowed to jump over players outside the coalition. This implies that an optimal order is such that the players in each component are rearranged optimally. Hence, for any coalition $T$,

$$
v(T)=\sum_{S \in T / \sigma_{0}} v(S)
$$

The following example illustrates a precedence sequencing game in case the precedence relation is a tree.

Example 2.1 Let $\left(N, \mathcal{P}, \sigma_{0}, p, \alpha\right)$ be a precedence sequencing situation, where $N=\{1,2,3,4\}, \mathcal{P}=$ $\{(1,2),(2,4),(1,3)\}, \sigma_{0}=(1,2,3,4), p=(1,1,1,1)$ and $\alpha=(1,2,3,4)$. Then the worth of the connected coalitions is $v(\{i\})=0$ for $i=1,2,3,4, v(\{1,2\})=0$, and $v(S)=1$ if $S=\{2,3\},\{3,4\}$, $\{1,2,3\},\{2,3,4\},\{1,2,3,4\}$. 
Note that (1) implies that precedence sequencing games are $\sigma_{0}$-component additive games, and, thus, balanced (cf. Curiel et al. (1994)). Recall that a game $(N, v)$ is called balanced if its core is non-empty. The core consists of all vectors that distribute $v(N)$, i.e., the revenues incurred when all players in $N$ cooperate, among the players in such a way that no subset of players can be better off by seceding from the rest of the players and acting on their own behalf. That is, a vector $x \in \mathbb{R}^{N}$ is in the core of a game $(N, v)$ if $\sum_{j \in N} x_{j}=v(N)$ and $\sum_{j \in S} x_{j} \geq v(S)$ for all $S \subset N$.

\section{Convexity of precedence sequencing games}

In this section we will establish the convexity of the precedence sequencing games corresponding to situations in which the precedence relations consist of parallel chains and the initial order is a concatenation of these chains.

The following example shows that precedence sequencing games that arise from a sequencing situation in which the precedence relation is a tree need not be convex. Recall that a game $(N, v)$ is called convex if for any $i, j \in N, i \neq j$ and any $S \subseteq N \backslash\{i, j\}$ it holds

$$
v(S \cup\{i, j\})-v(S \cup\{i\})-v(S \cup\{j\})+v(S) \geq 0 .
$$

Example 3.1 Consider the precedence sequencing game of Example 2.1. Then

$$
v(\{2,3,4\})-v(\{2,3\})-v(\{3,4\})+v(\{3\})=-1<0,
$$

which implies that $(N, v)$ is not convex.

Let $\left(N, \mathcal{P}, \sigma_{0}, p, \alpha\right)$ be a precedence sequencing situation. Then $\mathcal{P}$ is said to be a network of parallel chains if each player precedes at most one player and is preceded by at most one player, i.e., for each $i \in N$ it holds that $|\{j \in N:(i, j) \in \mathcal{P}\}| \leq 1$ and $|\{j \in N:(j, i) \in \mathcal{P}\}| \leq 1$. A chain is an ordered set of players $\left(i_{1}, \ldots, i_{k}\right)$ for which $\left(i_{l}, i_{l+1}\right) \in \mathcal{P}$ for each $l \in\{1, \ldots, k-1\}$ and for which there does not exist a player $j \in N$ such that $\left(j, i_{1}\right) \in \mathcal{P}$ or $\left(i_{k}, j\right) \in \mathcal{P}$.

Let $\left(N, \mathcal{P}, \sigma_{0}, p, \alpha\right)$ be a precedence sequencing situation where $\mathcal{P}$ is a network of parallel chains, $1, \ldots, C$ say. The set of players in chain $c=1, \ldots, C$ is denoted by $P(c)$. The sets $P(c)(c=1, \ldots, C)$ define a partition of $N$. We assume that $\sigma_{0}$ is some concatenation of these chains, i.e., $P(c)$ is connected for all $c=1, \ldots, C$. Without loss of generality we assume that the order of the chains is $1, \ldots, C$. The following example illustrates a concatenation of chains.

Example 3.2 Let $\left(N, \mathcal{P}, \sigma_{0}, p, \alpha\right)$ be a precedence sequencing situation, where $N=\{1,2,3,4,5,6\}$, $\mathcal{P}=\{(1,2),(3,4),(4,5),(5,6)\}, p=(1,1,1,1,1,1)$, and $\alpha=(2,5,6,6,3,6)$. The only two possible initial orders are $(1,2,3,4,5,6)$ and $(3,4,5,6,1,2)$, because $P(1)=\{1,2\}$ and $P(2)=\{3,4,5,6\}$.

For determining the precedence sequencing game corresponding to a sequencing situation in which the precedence relation is a concatenation of chains, we need an optimal order for each coalition. Therefore, we need the following additional notations and definitions. For any $T \subseteq N, T \neq \emptyset$, we define

$$
\alpha(T):=\sum_{i \in T} \alpha_{i}, \quad p(T):=\sum_{i \in T} p_{i}, \quad u(T):=\frac{\alpha(T)}{p(T)}
$$


where $u(T)$ is called the urgency index of coalition $T$.

By the component additivity of the precedence games (see (1)), we can restrict ourselves to calculating the worth of connected coalitions. Let $S$ be a connected coalition. Then there are chains $c$ and $c+k$ such that $S \cap P(c+l) \neq \emptyset$ for all $l=0, \ldots, k$ and $S \cap P(c-1)=S \cap P(c+k+1)=\emptyset$. For any $l=0, \ldots, k$, let $c h_{l}(S)=S \cap P(c+l)=\left\{i_{1}^{l}, \ldots, i_{n_{l}}^{l}\right\}$ be the (non-empty) intersection of $S$ with the players of chain $c+l$. Each $c h_{l}(S)$ owns in a natural way the ordering induced by $\sigma_{0}$, i.e., for $c h_{l}(S)$ it holds that $\sigma_{0}\left(i_{1}^{l}\right)<\sigma_{0}\left(i_{2}^{l}\right)<\ldots<\sigma_{0}\left(i_{n_{l}}^{l}\right)$. Note that $c h_{l}(S)=P(c+l)$ for all $l=1, \ldots, k-1$.

Before stating Sidney's algorithm, we introduce the concepts of heads and tails. A head of a chain $c=\left(i_{1}, \ldots, i_{k}\right)$ is a set $T \subseteq P(c)$ such that $T=\left\{i_{1}, \ldots, i_{l}\right\}$. Similarly, a tail of $c$ is a set $T \subseteq P(c)$ such that $T=\left\{i_{l}, \ldots, i_{k}\right\}$.

Now Sidney's algorithm provides a way to calculate an optimal order of the members of $S$ given precedence relations that consist of parallel chains and an initial order that is a concatenation of chains.

\section{Procedure: Optimal order of connected $S$}

\section{Step 1: Construction of Sidney-components}

For every $l=0, \ldots, k$, find the following coalitions:

$T_{1}^{l}:=\left\{i_{1}^{l}, \ldots, i_{t_{1}^{l}}^{l}\right\}$, the largest head of $c h_{l}(S)$ that satisfies

$$
u\left(\left\{i_{1}^{l}, \ldots, i_{t_{1}^{l}}^{l}\right\}\right)=\max _{1 \leq q \leq n_{l}} u\left(\left\{i_{1}^{l}, \ldots, i_{q}^{l}\right\}\right)
$$

For $m>1$

$$
\begin{array}{r}
T_{m}^{l}:=\left\{i_{t_{m-1}^{l}}^{l}+1\right. \\
\left.u, i_{t_{m}^{l}}^{l}\right\}, \text { the largest head of } c h_{l}(S) \backslash\left(\cup_{i=1}^{m-1} T_{i}^{l}\right) \text { that satisfies } \\
u\left(\left\{i_{t_{m-1}^{l}}^{l}+1, \ldots, i_{t_{m}^{l}}^{l}\right\}\right)=\max _{t_{m-1}^{l}+1 \leq q \leq n_{l}} u\left(\left\{i_{t_{m-1}^{l}}^{l}+1, \ldots, i_{q}^{l}\right\}\right) .
\end{array}
$$

Let $m_{l}$ be the number of sets we obtain in this way. Then, $\cup_{r=1, \ldots, m_{l}} T_{r}^{l}=c h_{l}(S)$. The sets $T_{r}^{l}$ $\left(l=0, \ldots, k\right.$ and $\left.r=1, \ldots, m_{l}\right)$ are called the Sidney-components of $S$.

\section{Step 2: Ordering Sidney-components}

Order the Sidney-components of $S$ in weakly decreasing order with respect to their urgency indices.

The following theorem follows from Sidney (1975).

Theorem 3.3 An order $\sigma^{S}$ that results from the procedure is admissible and optimal for $S$.

Example 3.4 Let $\left(N, \mathcal{P}, \sigma_{0}, p, \alpha\right)$ with $\sigma_{0}=(1,2,3,4,5,6)$ be defined as in Example 3.2. Let $S=$ $\{2,3,4,5,6\}$. Then $c h_{0}(S)=\{2\}$ and $c h_{1}(S)=\{3,4,5,6\}$. Following the first step of Sidney's algorithm we obtain $T_{1}^{0}=\{2\}, T_{1}^{1}=\{3,4\}$ and $T_{3}^{1}=\{5,6\}$, with $u(\{2\})=5, u(\{3,4\})=6$ and $u(\{5,6\})=4 \frac{1}{2}$, respectively. From the second step of the algorithm and Theorem 3.3 it follows that processing the jobs in the order $\sigma^{S}=(1,3,4,2,5,6)$ is optimal for coalition $S$ given the precedence relation $\mathcal{P}$. 
Let $(N, v)$ be the precedence sequencing game corresponding to $\left(N, \mathcal{P}, \sigma_{0}, p, \alpha\right)$. It follows from (1) and the optimality of $\sigma^{S} \in \Sigma(S, \mathcal{P})$ that $v(S)=(2 * 5+3 * 6+4 * 6+5 * 3+6 * 6)-(2 * 6+3 *$ $6+4 * 5+5 * 3+6 * 6)=2$.

The following lemmata describe relations between urgency indices, which facilitate the proof of our main result.

Lemma 3.5 Let $S, T \subset N$ be disjoint and non-empty. If $u(S) \geq u(T)$, then $u(S) \geq u(S \cup T) \geq u(T)$. If $u(S)=u(T)$, then $u(S)=u(S \cup T)=u(T)$.

Proof. Suppose $u(S) \geq u(T)$. It holds that $\frac{\alpha(S)}{p(S)}=u(S) \geq u(T)=\frac{\alpha(T)}{p(T)}$. Therefore $\alpha(S) p(T) \geq$ $\alpha(T) p(S)$. Adding $\alpha(S) p(S)$ or $\alpha(T) p(T)$ to both sides gives $\alpha(S)(p(S)+p(T)) \geq(\alpha(S)+\alpha(T)) p(S)$ and $(\alpha(S)+\alpha(T)) p(T) \geq \alpha(T)(p(S)+p(T))$, respectively. Hence, $u(S)=\frac{\alpha(S)}{p(S)} \geq \frac{\alpha(S)+\alpha(T)}{p(S)+p(T)}=$ $u(S \cup T)$ and $u(S \cup T)=\frac{\alpha(S \cup T)}{p(S \cup T)} \geq \frac{\alpha(T)}{p(T)}=u(T)$.

Now suppose $u(S)=u(T)$. Then it holds that $\alpha(S) p(T)=\alpha(T) p(S)$. Adding $\alpha(S) p(S)$ to both sides gives $\alpha(S)(p(S)+p(T))=(\alpha(S)+\alpha(T)) p(S)$, and equivalently, $u(S)=u(S \cup T)$.

Lemma 3.6 Let $S, T, W \subset N$ be disjoint and non-empty. If $u(W) \geq u(T) \geq u(S)$, then $u(S \cup T \cup W) \geq u(S \cup T)$.

Proof. Because $u(T) \geq u(S)$ it follows from Lemma 3.5 that $u(T) \geq u(S \cup T) \geq u(S)$, and therefore $u(W) \geq u(S \cup T)$. Applying Lemma 3.5 again gives $u(W) \geq u(S \cup T \cup W) \geq u(S \cup T)$.

Lemma 3.7 Let $T \subseteq N, T \neq \emptyset$ and let $T_{1}^{l}, \ldots, T_{m_{l}}^{l}$ be the Sidney-components of $T$ for some chain $l$. Then $u\left(T_{1}^{l}\right)>u\left(T_{2}^{l}\right)>\cdots>u\left(T_{m_{l}}^{l}\right)$.

Proof. Follows immediately from the definition of the Sidney-components and Lemma 3.5.

To prove our main result we need the following notation. For two coalitions $U, V \subseteq N$ with $U \cap V=$ $\emptyset$, we define ${ }^{2}$

$$
g(U, V):=(\alpha(V) p(U)-\alpha(U) p(V))_{+} .
$$

Note that $g(U, V) \geq 0$. For any two non-empty sets $U, V \subseteq N$ it holds that $g(U, V)>0$ if and only if $u(V)>u(U)$. Extending to two collections $\mathcal{U}, \mathcal{V} \subseteq 2^{N}$ with $U \cap V=\emptyset$ for each $U \in \mathcal{U}, V \in \mathcal{V}$, we define

$$
G(\mathcal{U}, \mathcal{V}):=\sum_{U \in \mathcal{U}, V \in \mathcal{V}} g(U, V)
$$

Theorem 3.8 Let $\left(N, \mathcal{P}, \sigma_{0}, p, \alpha\right)$ be a precedence sequencing situation where $\mathcal{P}$ is a network of parallel chains and $\sigma_{0}$ a concatenation of chains. Then the corresponding precedence sequencing game $(N, v)$ is convex.

\footnotetext{
${ }^{2}$ For $x \in \mathbb{R}$ we write $x_{+}=\max \{0, x\}$.
} 
Proof. The initial order is a concatenation of chains. Without loss of generality we assume that the order of the chains is $1,2, \ldots, C$. We have to show that (2) holds for every $i, j \in N, i \neq j$ and $S \subset N \backslash\{i, j\}$.

First suppose that $i$ and $j$ are in different components of $S \cup\{i, j\}$. Then applying (1) implies 2). Therefore we only consider situations in which $i$ and $j$ are in the same component of $S \cup\{i, j\}$. Because precedence games are $\sigma_{0}$-component additive, it is sufficient to consider situations where $S \cup\{i, j\}$ is connected. Without loss of generality assume that $\sigma_{0}(i)<\sigma_{0}(j)$. Now define (see Figure 1 for an illustration)

$$
\begin{aligned}
S_{1} & :=\left\{k \in S: \sigma_{0}(k)<\sigma_{0}(i)\right\}, \\
S_{2} & :=\left\{k \in S: \sigma_{0}(i)<\sigma_{0}(k)<\sigma_{0}(j)\right\}, \\
S_{3} & :=\left\{k \in S: \sigma_{0}(j)<\sigma_{0}(k)\right\} .
\end{aligned}
$$

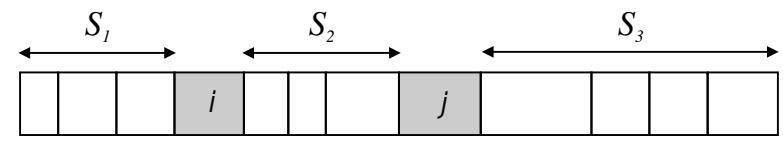

Figure 1: The sets $S_{1}, S_{2}$, and $S_{3}$

We distinguish between two cases.

CASE 1: $S_{1} \cup S_{3}=\emptyset$, i.e., $S=S_{2}$.

Suppose that $i$ and $j$ are in the same chain. In that case no reordering of the players is admissible, and therefore $v(S \cup\{i, j\})=v(S \cup\{j\})=v(S \cup\{i\})=v(S)=0$ and (2) holds. So now suppose that $i$ is an element of chain $c^{*}$ and $j$ is an element of chain $d^{*}$, where $c^{*}<d^{*}$. For convenience we introduce the following sets.

For $V=S \cup\{i, j\}, S \cup\{i\}$ let $C_{1}(V)$ be the collection of Sidney-components of $V$ that are contained in $c^{*}$ and that are not Sidney-components of $S \cup\{j\}$. Note that $C_{1}(S \cup\{i, j\})=C_{1}(S \cup\{i\})$, because $P\left(c^{*}\right) \cap(S \cup\{i, j\})=P\left(c^{*}\right) \cap(S \cup\{i\})$.

For $V=S \cup\{j\}, S$ let $C_{1}(V)$ be the collection of Sidney-components of $V$ that are contained in $c^{*}$ and that are not Sidney-components of $S \cup\{i, j\}$. Note that $C_{1}(S \cup\{j\})=C_{1}(S)$.

For $V=S \cup\{i, j\}, S \cup\{j\}$ let $C_{4}(V)$ be the collection of Sidney-components of $V$ that are contained in $d^{*}$ and that are not Sidney-components of $S \cup\{i\}$. Note that $C_{4}(S \cup\{i, j\})=C_{4}(S \cup\{j\})$.

For $V=S \cup\{i\}, S$ let $C_{4}(V)$ be the collection of Sidney-components of $V$ which are contained in $d^{*}$ and which are not Sidney-components of $S \cup\{i, j\}$. Note that $C_{4}(S \cup\{i\})=C_{4}(S)$.

See for an example Figure 2. Note that the end of $C_{1}$ and the beginning of $C_{4}$ coincide in all four situations. This follows straightforwardly from Lemma A.1 of the Appendix.

Moreover from Lemma A.2 it follows that

$$
v(S \cup\{i, j\})-v(S \cup\{i\})-v(S \cup\{j\})+v(S)
$$




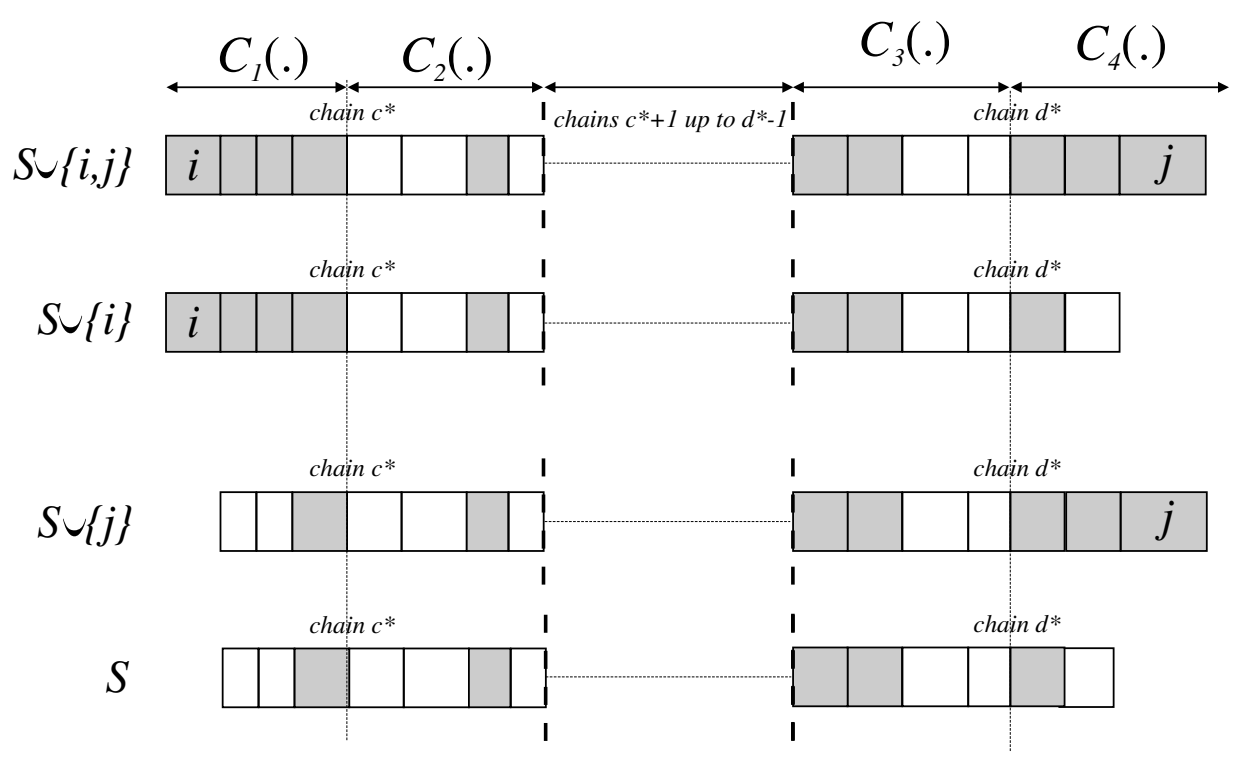

Figure 2: The sets $C_{1}($.$) up to C_{4}($.

$$
\begin{aligned}
= & G\left(C_{1}(S \cup\{i, j\}), C_{4}(S \cup\{i, j\})\right)-G\left(C_{1}(S \cup\{i\}), C_{4}(S \cup\{i\})\right) \\
& -G\left(C_{1}(S \cup\{j\}), C_{4}(S \cup\{j\})\right)+G\left(C_{1}(S), C_{4}(S)\right)
\end{aligned}
$$

From Lemma A.1 it follows that $C_{1}(S \cup\{i, j\})$ and $C_{4}(S \cup\{i, j\})$ contain only one element (i.e., Sidney-component). Let $U^{*}$ be the unique element of $C_{1}(S \cup\{i, j\})$ and let $V^{*}$ be the unique element of $C_{4}(S \cup\{i, j\})$. Substituting this in (4) we obtain

$$
\begin{aligned}
& v(S \cup\{i, j\})-v(S \cup\{i\})-v(S \cup\{j\})+v(S) \\
= & G\left(\left\{U^{*}\right\},\left\{V^{*}\right\}\right)-G\left(\left\{U^{*}\right\}, C_{4}(S \cup\{i\})\right) \\
& -G\left(C_{1}(S \cup\{j\}),\left\{V^{*}\right\}\right)+G\left(C_{1}(S), C_{4}(S)\right) \\
= & g\left(U^{*}, V^{*}\right)-\sum_{V \in C_{4}(S \cup\{i\})} g\left(U^{*}, V\right) \\
- & \sum_{U \in C_{1}(S \cup\{j\})} g\left(U, V^{*}\right)+\sum_{U \in C_{1}(S), V \in C_{4}(S)} g(U, V),
\end{aligned}
$$

where the second equality holds by (3). Hence, (2) is satisfied if expression (5) is nonnegative.

Subcase 1a: Suppose $g\left(U^{*}, V^{*}\right)=0$, i.e., $u\left(U^{*}\right) \geq u\left(V^{*}\right)$. Because $V^{*}$ is a Sidney-component, it follows from the definition of Sidney-components that $u\left(V^{*}\right) \geq u\left(V_{1}\right)$, where $V_{1}$ is the first Sidneycomponent in $C_{4}(S \cup\{i\})$. Hence, $u\left(U^{*}\right) \geq u\left(V_{1}\right)$, and $g\left(U^{*}, V_{1}\right)=0$. From Lemma 3.7 it follows that $\sum_{V \in C_{4}(S \cup\{i\})} g\left(U^{*}, V\right)=0$. Similarly, it can be shown that $\sum_{U \in C_{1}(S \cup\{j\})} g\left(U, V^{*}\right)=0$ and $\sum_{U \in C_{1}(S), V \in C_{4}(S)} g(U, V)=0$, and therefore expression (5) is nonnegative.

Subcase 1b: Suppose $g\left(U^{*}, V^{*}\right)>0$, i.e., $u\left(V^{*}\right)>u\left(U^{*}\right)$. Define 


$$
\begin{gathered}
V^{*}(a):=\cup_{V \in C_{4}(S \cup\{i\}): g\left(U^{*}, V\right)>0} V \\
V^{*}(b):=V^{*} \backslash V^{*}(a) .
\end{gathered}
$$

From Lemma 3.7 it follows that $V^{*}(a)$ is a head of $V^{*}$ that consist of the players of those Sidneycomponents of $C_{4}(S \cup\{i\})$ with higher urgency index than $U^{*}$. Note that $j \in V^{*}(b)$, and therefore $V^{*}(b) \neq \emptyset$. Similarly we define

$$
\begin{gathered}
U^{*}(b):=\cup_{U \in C_{1}(S \cup\{j\}): g\left(U, V^{*}\right)>0} U \\
U^{*}(a):=U^{*} \backslash U^{*}(b) .
\end{gathered}
$$

From Lemma 3.7 it follows that $U^{*}(b)$ is a tail of $U^{*}$ that consist of the players of those Sidneycomponents of $C_{1}(S \cup\{j\})$ with lower urgency index than $V^{*}$. Note that $i \in U^{*}(a)$ and therefore $U^{*}(a) \neq \emptyset$. Rewriting the first two terms of (5) we obtain

$$
\begin{aligned}
& g\left(U^{*}, V^{*}\right)-\sum_{V \in C_{4}(S \cup\{i\})} g\left(U^{*}, V\right) \\
= & g\left(U^{*}, V^{*}\right)-\sum_{V \in C_{4}(S \cup\{i\}): V \subseteq V^{*}(a)} g\left(U^{*}, V\right) \\
= & \alpha\left(V^{*}\right) p\left(U^{*}\right)-\alpha\left(U^{*}\right) p\left(V^{*}\right)-\sum_{V \in C_{4}(S \cup\{i\}): V \subseteq V^{*}(a)}\left(\alpha(V) p\left(U^{*}\right)-\alpha\left(U^{*}\right) p(V)\right) \\
= & \alpha\left(V^{*}\right) p\left(U^{*}\right)-\alpha\left(U^{*}\right) p\left(V^{*}\right)-\alpha\left(V^{*}(a)\right) p\left(U^{*}\right)+\alpha\left(U^{*}\right) p\left(V^{*}(a)\right) \\
= & \alpha\left(V^{*}(b)\right) p\left(U^{*}\right)-\alpha\left(U^{*}\right) p\left(V^{*}(b)\right),
\end{aligned}
$$

where the second equality follows from $u\left(V^{*}\right)>u\left(U^{*}\right)$ and $u(V)>u\left(U^{*}\right)$ for all $V \in C_{4}(S \cup\{i\})$ with $V \subseteq V^{*}(a)$. Rewriting the last two terms of (5) we obtain

$$
\begin{aligned}
& \sum_{U \in C_{1}(S \cup\{j\})} g\left(U, V^{*}\right)-\sum_{U \in C_{1}(S), V \in C_{4}(S)} g(U, V) \\
\leq & \sum_{U \in C_{1}(S \cup\{j\}): U \subseteq U^{*}(b)} g\left(U, V^{*}\right)-\sum_{U \in C_{1}(S), V \in C_{4}(S): U \subseteq U^{*}(b), V \subseteq V^{*}(a)} g(U, V) \\
\leq & \sum_{U \in C_{1}(S \cup\{j\}): U \subseteq U^{*}(b)}\left(\alpha\left(V^{*}\right) p(U)-\alpha(U) p\left(V^{*}\right)\right) \\
& -\sum_{U \in C_{1}(S), V \in C_{4}(S): U \subseteq U^{*}(b), V \subseteq V^{*}(a)}(\alpha(V) p(U)-\alpha(U) p(V)) \\
= & \alpha\left(V^{*}\right) p\left(U^{*}(b)\right)-\alpha\left(U^{*}(b)\right) p\left(V^{*}\right)-\alpha\left(V^{*}(a)\right) p\left(U^{*}(b)\right)+\alpha\left(U^{*}(b)\right) p\left(V^{*}(a)\right) \\
= & \alpha\left(V^{*}(b)\right) p\left(U^{*}(b)\right)-\alpha\left(U^{*}(b)\right) p\left(V^{*}(b)\right) .
\end{aligned}
$$

The first inequality follows from the definition of $U^{*}(b)$. The second inequality follows from $g(U, V) \geq$ $\alpha(V) u(U)-\alpha(U) p(V)$ for all $U, V \subseteq N$.

Substituting (6) and (7) in (5) we obtain 


$$
\begin{aligned}
& v(S \cup\{i, j\})-v(S \cup\{i\})-v(S \cup\{j\})+v(S) \\
\geq \quad & \alpha\left(V^{*}(b)\right) p\left(U^{*}(a)\right)-\alpha\left(U^{*}(a)\right) p\left(V^{*}(b)\right) .
\end{aligned}
$$

To show that expression (8) is nonnegative, we will prove that $u\left(V^{*}(b)\right) \geq u\left(V^{*}\right)$ and $u\left(U^{*}\right) \geq$ $u\left(U^{*}(a)\right)$. This implies, using the assumption $u\left(V^{*}\right)>u\left(U^{*}\right)$, that $u\left(V^{*}(b)\right)>u\left(U^{*}(a)\right)$. As a result expression (8) is nonnegative.

Suppose that $V^{*}(a)=\emptyset$, then $V^{*}(b)=V^{*}$ and hence $u\left(V^{*}(b)\right)=u\left(V^{*}\right)$. So suppose that $V^{*}(a) \neq \emptyset$ and suppose that $u\left(V^{*}(a)\right)>u\left(V^{*}(b)\right)$. Then using Lemma 3.5 it follows that $u\left(V^{*}(a)\right)>u\left(V^{*}\right)>$ $u\left(V^{*}(b)\right)$. This implies that $V^{*}$ is not a Sidney-component of $S \cup\{i, j\}$, which is a contradiction. Hence, $u\left(V^{*}(b)\right) \geq u\left(V^{*}(a)\right)$ and using Lemma 3.5 it follows that $u\left(V^{*}(b)\right) \geq u\left(V^{*}\right)$. The proof that $u\left(U^{*}\right) \geq u\left(U^{*}(a)\right)$ runs similarly.

CASE 2: $S_{1} \cup S_{3} \neq \emptyset$.

First suppose that $S=S_{2} \cup S_{3}$, i.e., $S_{1}=\emptyset$. Let $S_{3}=\left\{h_{1}, \ldots, h_{q}\right\}$ where $\sigma_{0}\left(h_{1}\right)<\cdots<\sigma_{0}\left(h_{q}\right)$. Then

$$
\begin{aligned}
& v(S \cup\{i, j\})-v(S \cup\{i\})-v(S \cup\{j\})+v(S) \\
= & v\left(S_{2} \cup S_{3} \cup\{i, j\}\right)-v\left(S_{2} \cup S_{3} \cup\{i\}\right)-v\left(S_{2} \cup S_{3} \cup\{j\}\right)+v\left(S_{2} \cup S_{3}\right) \\
= & v\left(S_{2} \cup S_{3} \cup\{i, j\}\right)-\left(v\left(S_{2} \cup\{i\}\right)+v\left(S_{3}\right)\right)-v\left(S_{2} \cup S_{3} \cup\{j\}\right)+\left(v\left(S_{2}\right)+v\left(S_{3}\right)\right) \\
= & v\left(S_{2} \cup S_{3} \cup\{i, j\}\right)-v\left(S_{2} \cup\{i\}\right)-v\left(S_{2} \cup S_{3} \cup\{j\}\right)+v\left(S_{2}\right) \\
= & v\left(S_{2} \cup\{i, j\}\right)-v\left(S_{2} \cup\{i\}\right)-v\left(S_{2} \cup\{j\}\right)+v\left(S_{2}\right) \\
+ & v\left(S_{2} \cup S_{3} \cup\{i, j\}\right)-v\left(S_{2} \cup\{i, j\}\right)-v\left(S_{2} \cup S_{3} \cup\{j\}\right)+v\left(S_{2} \cup\{j\}\right)
\end{aligned}
$$

where the second equality holds because $S_{2} \cup S_{3} \cup\{i\}$ and $S_{2} \cup S_{3}$ are disconnected. We will show that expression (9) as well as expression (10) is nonnegative.

From Case 1 it follows that

$$
v\left(S_{2} \cup\{i, j\}\right)-v\left(S_{2} \cup\{i\}\right)-v\left(S_{2} \cup\{j\}\right)+v\left(S_{2}\right) \geq 0,
$$

which shows that expression (9) is nonnegative.

Now let $T_{1}=S_{2} \cup\{j\}$, and for $l \in\{2, \ldots, q\}$ let $T_{l}=S_{2} \cup\left\{j, h_{1}, \ldots, h_{l-1}\right\}$. From Case 1 it follows that for each $l \in\{1, \ldots, q\}$

$$
v\left(T_{l} \cup\left\{i, h_{l}\right\}\right)-v\left(T_{l} \cup\{i\}\right)-v\left(T_{l} \cup\left\{h_{l}\right\}\right)+v\left(T_{l}\right) \geq 0 .
$$

Now it holds that

$$
\sum_{l=1}^{q}\left(v\left(T_{l} \cup\left\{i, h_{l}\right\}\right)-v\left(T_{l} \cup\{i\}\right)-v\left(T_{l} \cup\left\{h_{l}\right\}\right)+v\left(T_{l}\right)\right)
$$




$$
\begin{aligned}
& =\sum_{l=1}^{q}\left(v\left(T_{l} \cup\left\{i, h_{l}\right\}\right)-v\left(T_{l} \cup\{i\}\right)\right)+\sum_{l=1}^{q}\left(-v\left(T_{l} \cup\left\{h_{l}\right\}\right)+v\left(T_{l}\right)\right) \\
& =\left(v\left(T_{q} \cup\left\{i, h_{q}\right\}\right)-v\left(T_{1} \cup\{i\}\right)\right)+\left(-v\left(T_{q} \cup\left\{h_{q}\right\}\right)+v\left(T_{1}\right)\right) \\
& =v\left(S_{2} \cup S_{3} \cup\{i, j\}\right)-v\left(S_{2} \cup\{i, j\}\right)-v\left(S_{2} \cup S_{3} \cup\{j\}\right)+v\left(S_{2} \cup\{j\}\right) \geq 0,
\end{aligned}
$$

which shows that expression (10) is nonnegative. Hence (2) holds if $S_{3} \neq \emptyset$ and $S_{1}=\emptyset$. A similar argument shows that (2) holds if $S_{1}$ and $S_{3}$ are both non-empty.

Finally we illustrate that convexity is lost if the initial order is not a concatenation of chains.

Example 3.9 Let us consider the precedence sequencing situation $\left(N, \mathcal{P}, \sigma_{0}, p, \alpha\right)$ given by $N=\{1,2,3\}$, $\mathcal{P}=\{(1,3)\}, \sigma_{0}=(1,2,3), p=(1,1,1)$, and $\alpha=(1,2,3)$. Hence, $\sigma_{0}$ is not a concatenation of chains. Let $(N, v)$ be the corresponding precedence sequencing game. It can easily be verified that

$$
v(\{1,2,3\})-v(\{2,3\})-v(\{1,2\})+v(\{2\})=1-1-1+0<0 .
$$

So $(N, v)$ is not convex.

\section{Appendix}

Lemma A.1 Let $\left(N, \mathcal{P}, \sigma_{0}, \alpha, p\right)$ be a precedence sequencing situation with $\mathcal{P}$ a network of parallel chains and let $\sigma_{0}$ be a concatenation of chains. The sets $C_{1}(S \cup\{i, j\})$ and $C_{4}(S \cup\{i, j\})$ contain precisely one element (i.e., Sidney-component).

Proof. We will show that $C_{1}(S \cup\{i, j\})$ contains a single element. If $i$ is the only player in $P\left(c^{*}\right) \cap$ $(S \cup\{i, j\})$, then $C_{1}(S \cup\{i, j\})=\{\{i\}\}$. So assume that $i$ is not the only player in $P\left(c^{*}\right) \cap(S \cup\{i, j\})$ and suppose that the Sidney-component of $S \cup\{i, j\}$ containing $i$ is $\{i\} \cup_{l=1}^{m-1} A_{l} \cup B$, where $A_{l}$ is a Sidney-component of $S \cup\{j\}$ for each $l \in\{1, \ldots, m\}$ and where $B$ is a proper head of $A_{m}$, i.e., $B \neq \emptyset$ and $B \neq A_{m}$. Then it holds that $u\left(\{i\} \cup_{l=1}^{m-1} A_{l} \cup B\right) \geq u\left(\{i\} \cup_{l=1}^{m-1} A_{l}\right)$. Now suppose that $u(B)<u\left(\{i\} \cup_{l=1}^{m-1} A_{l}\right)$. Then from Lemma 3.5 it follows that $u\left(\{i\} \cup_{l=1}^{m-1} A_{l} \cup B\right)<u\left(\{i\} \cup_{l=1}^{m-1} A_{l}\right)$, which is a contradiction. Hence, $u(B) \geq u\left(\{i\} \cup_{l=1}^{m-1} A_{l}\right)$.

Because $A_{m}$ is a Sidney-component of $S \cup\{j\}$, it holds that $u\left(A_{m} \backslash B\right) \geq u(B)$. Hence, we have $u\left(A_{m} \backslash B\right) \geq u(B) \geq u\left(\{i\} \cup_{l=1}^{m-1} A_{l}\right)$. From Lemma 3.6, by using $S=\{i\} \cup_{l=1}^{m-1} A_{l}, T=B$ and $W=A_{m} \backslash B$, we obtain that $u\left(\{i\} \cup_{l=1}^{m} A_{l}\right) \geq u\left(\{i\} \cup_{l=1}^{m-1} A_{l} \cup B\right)$, which is a contradiction to the assumption that the Sidney-component of $S \cup\{i, j\}$ containing $i$ is $\{i\} \cup_{l=1}^{m-1} A_{l} \cup B$. Therefore, the Sidney-component of $S \cup\{i, j\}$ containing $i$ is of the form $\{i\} \cup_{l=1}^{m} A_{l}$, and we conclude that $C_{1}(S \cup\{i, j\})$ contains a single element. Similarly it can be shown that $C_{4}(S \cup\{i, j\})$ contains one element.

Lemma A.2 Let $\left(N, \mathcal{P}, \sigma_{0}, \alpha, p\right)$ be a precedence sequencing situation with $\mathcal{P}$ a network of parallel chains let $\sigma_{0}$ be a concatenation of chains. Let $(N, v)$ be the corresponding precedence sequencing 
game. It holds that

$$
\begin{aligned}
& v(S \cup\{i, j\})-v(S \cup\{i\})-v(S \cup\{j\})+v(S) \\
= & G\left(C_{1}(S \cup\{i, j\}), C_{4}(S \cup\{i, j\})\right)-G\left(C_{1}(S \cup\{i\}), C_{4}(S \cup\{i\})\right) \\
& -G\left(C_{1}(S \cup\{j\}), C_{4}(S \cup\{j\})\right)+G\left(C_{1}(S), C_{4}(S)\right) .
\end{aligned}
$$

Proof. Besides the already introduced sets $C_{1}(V)$ and $C_{4}(V)$, where $V=S \cup\{i, j\}, S \cup\{i\}, S \cup\{j\}, S$, we introduce the following collections of Sidney-components (for an illustration see Figure 2). For $V=S \cup\{i, j\}, S \cup\{i\}$ let $C_{2}(V)$ be the collection of Sidney-components of $V$ that are contained in $c^{*}$ and that are also Sidney-components of $S \cup\{j\}$.

For $V=S \cup\{j\}, S$ let $C_{2}(V)$ be the collection of Sidney-components of $V$ that are contained in $c^{*}$ and that are also Sidney-components of $S \cup\{i, j\}$. Note that $C_{2}(S \cup\{i, j\})=C_{2}(S \cup\{i\})=$ $C_{2}(S \cup\{j\})=C_{2}(S)$.

For $V=S \cup\{i, j\}, S \cup\{j\}$ let $C_{3}(V)$ be the collection of Sidney-components of $V$ that are contained in $d^{*}$ and that are also Sidney-components of $S \cup\{i\}$.

For $V=S \cup\{i\}, S$ let $C_{3}(V)$ be the collection of Sidney-components of $V$ that are contained in $d^{*}$ and that are also Sidney-components of $S \cup\{i, j\}$. Note that $C_{3}(S \cup\{i, j\})=C_{3}(S \cup\{i\})=$ $C_{3}(S \cup\{j\})=C_{3}(S)$.

For $l \in\left\{c^{*}+1, \ldots, d^{*}-1\right\}$ let $D_{l}$ be the collection of Sidney-components that are contained in chain $l$.

Finally, for $V=S \cup\{i, j\}, S \cup\{i\}, S \cup\{j\}, S$ let $C_{12}(V)=C_{1}(V) \cup C_{2}(V)$ and let $C_{34}(V)=$ $C_{3}(V) \cup C_{4}(V)$.

For $T=S \cup\{i, j\}, S \cup\{i\}, S \cup\{j\}, S$ it holds that

$$
\begin{aligned}
v(T) & =\sum_{l=c^{*}+1}^{d^{*}-1}\left[G\left(C_{12}(T), D_{l}\right)+G\left(C_{12}(T), C_{34}(T)\right)\right] \\
& +\sum_{l, m \in\left\{c^{*}+1, \ldots, d^{*}-1\right\}: l<m} G\left(D_{l}, D_{m}\right)+\sum_{l=c^{*}+1}^{d^{*}-1} G\left(D_{l}, C_{34}(T)\right) .
\end{aligned}
$$

Now it is straightforward, using $C_{12}(S \cup\{i, j\})=C_{12}(S \cup\{i\}), C_{12}(S \cup\{j\})=C_{12}(S)$, $C_{34}(S \cup\{i, j\})=C_{34}(S \cup\{j\})$ and $C_{34}(S \cup\{i\})=C_{34}(S)$, to show that

$$
\begin{aligned}
& v(S \cup\{i, j\})-v(S \cup\{i\})-v(S \cup\{j\})+v(S) \\
= & G\left(C_{12}(S \cup\{i, j\}), C_{34}(S \cup\{i, j\})\right)-G\left(C_{12}(S \cup\{i\}), C_{34}(S \cup\{i\})\right) \\
& -G\left(C_{12}(S \cup\{j\}), C_{34}(S \cup\{j\})\right)+G\left(C_{12}(S), C_{34}(S)\right) . \\
= & G\left(C_{1}(S \cup\{i, j\}), C_{4}(S \cup\{i, j\})\right)-G\left(C_{1}(S \cup\{i\}), C_{4}(S \cup\{i\})\right) \\
& -G\left(C_{1}(S \cup\{j\}), C_{4}(S \cup\{j\})\right)+G\left(C_{1}(S), C_{4}(S)\right),
\end{aligned}
$$

which proves the lemma. 


\section{References}

Borm, P., Fiestras-Janeiro, G., Hamers, H., Sánchez, E., and Voorneveld, M. (2002). "On the Convexity of Sequencing Games with Due Dates," European Journal of Operational Research, 136, 616-634.

Calleja, P., Borm, P., Hamers, H., Klijn, F., and Slikker, M. (2002). "On a New Class of Parallel Sequencing Situations and Related Games,” Annals of Operations Research, 109, 265-277.

Curiel, I., Pederzoli, G., and Tijs, S. (1989). "Sequencing Games," European Journal of Operational Research, 40, 344-351.

Curiel, I., Potters, J., Rajendra Prasad, V., Tijs, S., Veltman, B. (1994). "Sequencing and Cooperation," Operations Research, 42, 566-568.

Hamers, H., Borm, P., and Tijs, S. (1995). "On Games corresponding to Sequencing Situations with Ready Times," Mathematical Programmming, 70, 1-13.

Hamers, H., Suijs, J., Tijs, S., and Borm, P. (1996). “The Split Core for Sequencing Games," Games and Economic Behavior, 15, 165-176.

Hamers, H., Klijn, F., and Suijs, J. (1999). "On the Balancedness of Multiple Machine Sequencing Games," European Journal of Operational Research, 119, 678-691.

Ichiishi, T. (1981). "Super-Modularity: Applications to Convex Games and the Greedy Algorithm for LP,” Journal of Economic Theory, 25, 283-286.

Maschler, M., Peleg, B., and Shapley, L. (1972). “The Kernel and Bargaining Set of Convex Games," International Journal of Game Theory, 2, 73-93.

Nouweland van den, A., Krabbenborg, M., and Potters, J. (1992). "Flowshops with a Dominant Machine," European Journal of Operational Research, 62, 38-46.

Shapley, L. (1971). “Cores of Convex Games,” International Journal of Game Theory, 1, 11-26.

Sidney, J. (1975). "Decomposition Algorithms for Single-Machine Sequencing with Precedence Relations and Deferral Costs," Operations Research, 23, 283-298.

Tijs, S. (1981). "Bounds for the Core and the $\tau$-value" In: Game Theory and Mathematical Economics (Eds. Moeschlin O. and Pallaschke P.), North Holland Publishing Company, pp. 123-132.

Velzen van, B. and Hamers, H. (2002). "On the Balancedness of Relaxed Sequencing Games," CentER Discussion Paper 2002-49, Tilburg University, The Netherlands (to appear in Mathematical Methods of Operations Research). 